\title{
On Sum - Throughput and Fairness Index analysis of Downlink NOMA Over OFDMA for Machine - to - Machine Communication
}

\author{
Selvam $K^{1}$, Kumar $K^{2}$ \\ Research Scholar, Department of ECE, Pondicherry Engineering College, Puducherry, India ${ }^{1}$ \\ Professor, Department of ECE, Pondicherry Engineering College, Puducherry, India ${ }^{2}$
}

\begin{abstract}
With the anticipated exponential increase in the volume of cellular traffic e.g., beyond a 1000 fold increase in the next decade, significant gains in sum throughput and Fairness index of the system are required to satisfy all the users with the excellent quality of user experience. As a potential candidate of multiple access technique for 5G, NonOrthogonal Multiple Access (NOMA) can be an effective and noteworthy solution to M2M communications to serve more number of users by the same resource to achieve significant spectrum efficiency gain over conventional Orthogonal frequency division multiple access. NOMA, a novel technique can provide more satisfied level of Sumthroughput and Fairness index value to all the devices and users. In this paper, the performance of Sum-throughput and Fairness Index of NOMA technique is analysed and compared it with existing OFDMA system. Simulation results demonstrate that the NOMA technique outperforms the prevailing OFDMA technique with respect to Sum-throughput and Fairness Index of users, thus can be a potential solution for effective use of limited bandwidth constraint to suffix the need of future M2M communications.
\end{abstract}

Keywords: Sum - Throughput, Fairness Index, 5G Networks, Machine - to - Machine (M2M) Communications, NonOrthogonal Multiple Access (NOMA), Orthogonal Frequency Multiple Access Technique (OFDMA)

\section{INTRODUCTION}

Recently, an exponential growth of M2M devices was witnessed with more than half a billion devices getting added to the network in the year 2016. In addition to the existing traffic, it is being predicted that there will be 50 billion Internet of Things (IoT) devices getting connected to Internet by the year 2020. The drastic increase in the number of mobile devices and M2M Devices are expected to create a huge data traffic in the network in the future [1-3]. Orthogonal Frequency Division Multiple Access, a key successful technology up to fourth generation cellular networks are unable to meet the huge demand of higher spectrum efficiency, higher data traffic, sum-throughput and user fairness of the system. Hence a key technology is required utmost to solve the current requirements of huge data traffic. NonOrthogonal Multiple Access (NOMA), a novel multiple access technology is considered as a capable and most suitable multiple access technology for the future generation cellular networks due to such a high Spectral efficiency and massive connectivity of NOMA [4-7]. Non-Orthogonal Multiple Access (NOMA) defines multiuser transmission schemes which allows interference among users. The concept of NOMA with power control and Successive Interference Cancelation (SIC) was introduced. In NOMA, same frequency resource can be assigned to multiple users. This feature enables NOMA to provide higher spectral efficiency, massive connectivity required by M2M service and to address huge data traffic for future cellular networks[8-9].

In downlink power-domain Non-Orthogonal Multiple Access (NOMA), the base station transmits signals to more number of users at a single resource (time and frequency) by allocating a different power to each signal. At the receiver end, each User Equipment (UE) separates its own signal from the superimposed signal with successive interference cancellation so that Spectral Efficiency (SE) can be enhanced. The user with poor channel condition considers other user's messages as interference while the user with better channel condition uses SIC before decoding its own information [10-13].

Recently, researchers have been extensively working on Non-orthogonal Multiple access technology to effectively utilize its potential to the emerging need of Machine-to-Machine Communication (M2M) services on achieving higher sum throughput and excellent fairness index values for devices and users [14-15]. In [16-17], A minimizationmaximization method is analysed to optimize sum rate in the downlink Non-orthogonal multiple access. User fairness is also extensively studied and optimum method is proposed to enhance its value in NOMA system [18]. In order to reduce the complexity of NOMA system and to enhance Spectral efficiency and Massive connectivity of M2M devices, 
the impact of user pairing in 5G system is studied in [19]. In [20-21], the Trade-off between Throughput and Fairness index values are studied for Non orthogonal multiple access(NOMA) system.

In this paper, Sum-throughput and Fairness-index values of NOMA are analysed and studied. The results are then compared with the Sum-throughput and Fairness-index values of conventional orthogonal multiple access (OFDMA) technique to prove the excellent potential of NOMA for serving M2M devices.. The remaining of this paper is discussed as given below. Section II discusses about the System model of NOMA and OFDMA techniques. The Sumthroughput and fairness index values of NOMA and OFDMA are also extensively analysed in Section II. Simulation results are extensively presented in Section III. The conclusion part of the paper is given in Section IV.

\section{SYSTEM MODEL}

This section presents the basic model and concepts of Two user Non-orthogonal multiple access system in which a Base station sits at the centre and the two users are randomly distributed. Two cellular users namely User1(UE1) and User2(UE2) are located in such a way that one of the user is closer to Base station and the other user is situated far away from the Base station. Two cellular users access the network by means of Non-orthogonal multiple access or OFDMA. The system model of downlink NOMA is considered in such a way that superposition coding is executed at the Base station and the Successive interference cancellation is performed at the received terminals to decode the user information which can be illustrated in the figure 1. In NOMA system, SC is used for superimposing multiple users signals over the same RB in power domain. Hence, SC is effectively increasing the capacity of NOMA system without expanding the bandwidth which is a crucial demand of M2M service.
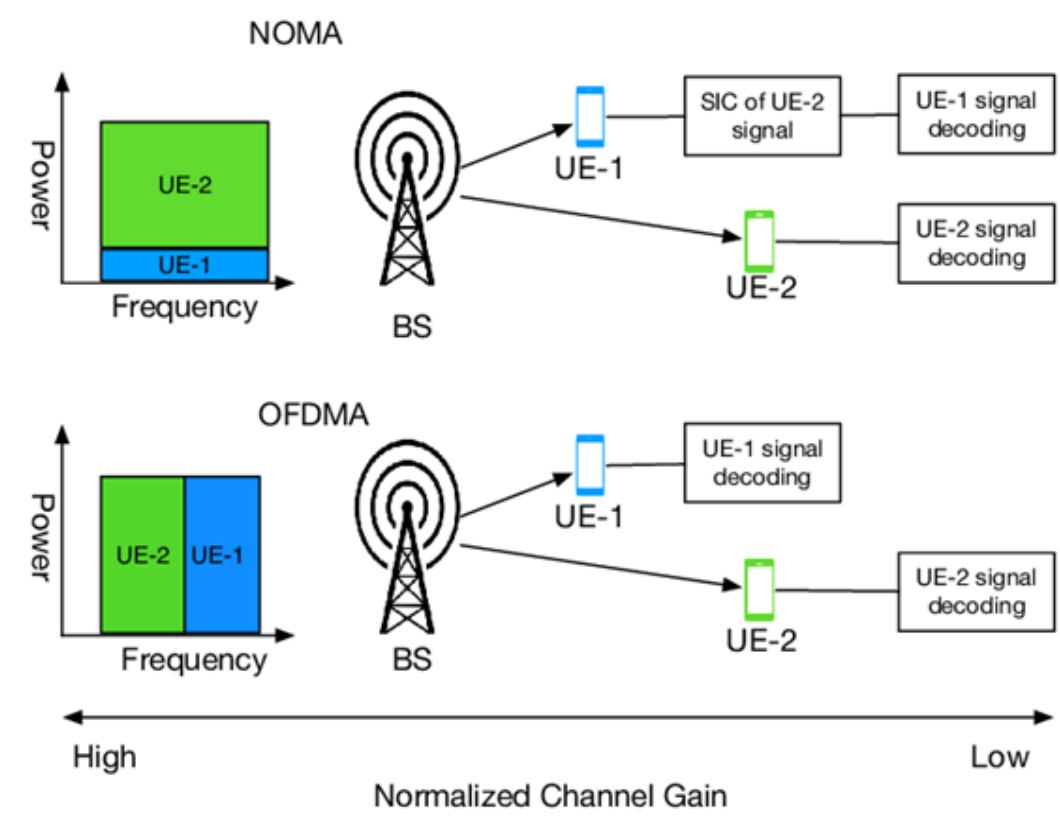

Fig. 1 (a) Downlink NOMA for 2 user case (b) Downlink OFDMA for 2 user case

The Base station transmits the information to both the users with different power allocation p1 and p2 on the same subcarrier, respectively. Power allocation to the users are decided based on the distance of users from Base station(BS). In NOMA system model, the users which are closer to the BS are allocated with less power and users which are far away from BS are assigned with higher power. For NOMA, both the users utilize the entire allocated bandwidth fully. But in OFDMA, the bandwidth is shared between the users. At the receiver terminal of user2 which is situated far away from $\mathrm{BS}$, information is decoded directly from received signal. The user which is closer to BS uses SIC(successive interference cancellation) technique to cancel other user information and then decode its own signal.

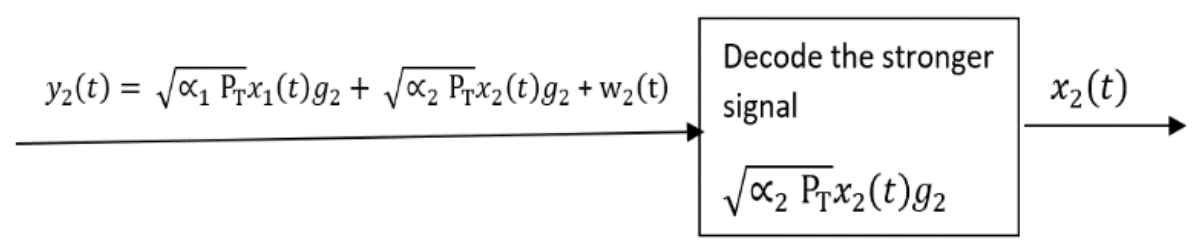


Vol. 8, Issue 5, May 2019

The mechanism behind SIC is that the user with weak channel conditions considers the signal of the user with the better channel as noise. Then it decodes its own data from the received signal. With respect to other side, the user with the good channel condition performs SIC and then it decodes the data of the weaker user. Finally it deducts it from the received signal and decode its own data. The concept of NOMA with SIC receiver is illustrated in figure 2.

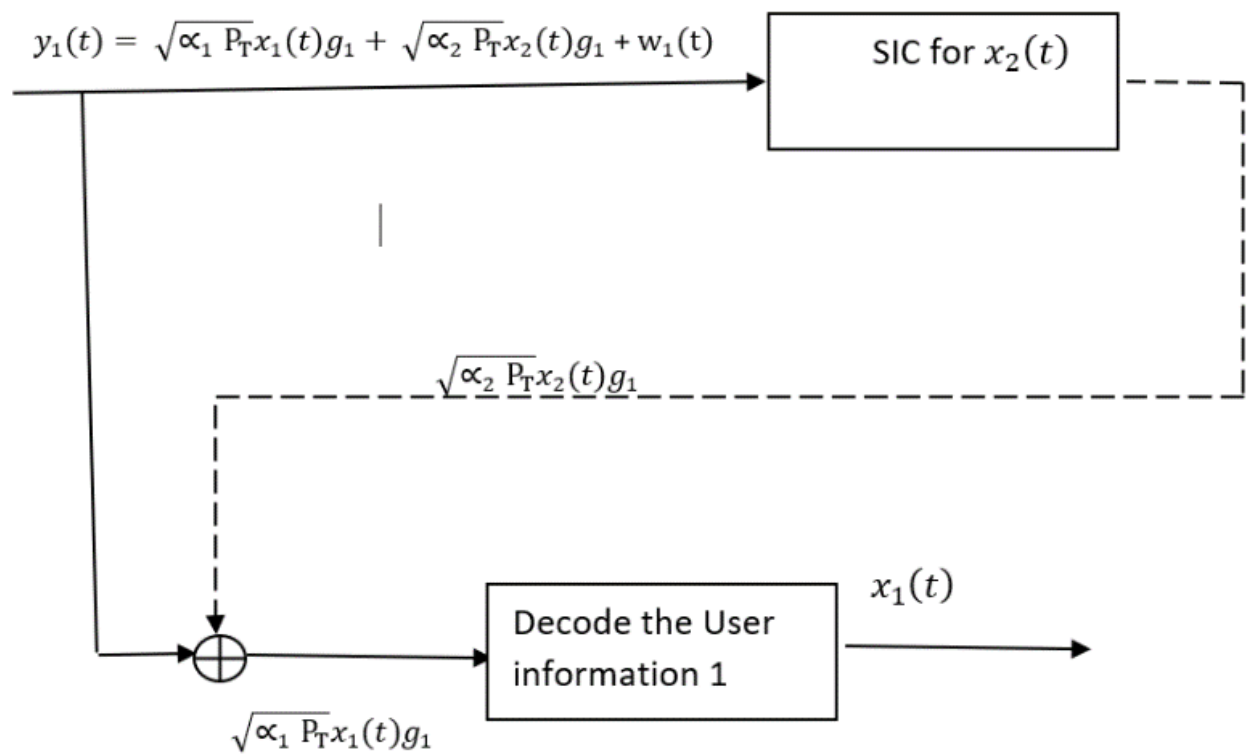

Fig. 2 Block diagram of NOMA decoders at the receiving terminals

Considering a downlink transmission scenario where a single base station transmits superposition coded information to the users in the cell.

The transmitted signal by the BS is expressed as

$$
x(t)=\sum_{k=1}^{K} \sqrt{\alpha_{k} P_{T}} x_{k}(t)
$$

Where $\propto_{k}$ denotes power allocation corresponding to $\mathrm{UE}_{\mathrm{k}}, \mathrm{x}_{\mathrm{k}}(\mathrm{t})$ represents information signal and $\mathrm{P}_{\mathrm{T}}$ denotes the total available power at the BS. In NOMA, the power allocated to each user equipment $\mathrm{UE}_{k}$ then becomes $\mathrm{P}_{k}=\propto_{k} \mathrm{P}_{\mathrm{T}}$. The fraction of powers are allocated to the users based on the distance of User Equipment's to the Base station. The closest user has least power allocation and the farthest user is allocated with more power.

The received signal corresponding to $\mathrm{UE}_{\mathrm{k}}$ is

$$
y_{k}(t)=x(t) g_{k}+w_{k}(t)
$$

Where $w_{k}(t)$ represents additive white gaussian noise with mean zero and noise density N0 (W/Hz) and $g_{k}$ represents the channel attenuation factor between the $\mathrm{UE}_{\mathrm{k}}$ and the $\mathrm{BS}$.

For NOMA, The throughput for every user equipment is defined as

$$
\mathrm{R}_{\mathrm{k}}=\mathrm{W} \log _{2}\left[1+\frac{\mathrm{P}_{\mathrm{k}} \mathrm{g}_{\mathrm{k}}^{2}}{\mathrm{~N}_{0} \mathrm{~W}+\sum_{\mathrm{i}=1}^{\mathrm{k}-1} \mathrm{P}_{\mathrm{i}} \mathrm{g}_{\mathrm{k}}^{2}}\right]
$$

Where W depicts system transmission bandwidth. For OFDMA, User equipment's are allocated to a collection of subcarriers for receiving the data. In OFDMA, Users share the total bandwidth and power equally.

Hence the throughput corresponding to individual User equipment with respect to OFDMA system can be written as

$$
\mathrm{R}_{\mathrm{k}}=\mathrm{W}_{\mathrm{k}} \log _{2}\left[1+\frac{\mathrm{P}_{\mathrm{k}} \mathrm{g}_{\mathrm{k}}^{2}}{\mathrm{~N}_{\mathrm{k}}}\right]
$$


where $\mathrm{W}_{\mathrm{k}}=\frac{\mathrm{w}}{\mathrm{K}}$ and $\mathrm{N}_{\mathrm{k}}=\mathrm{N}_{0} \mathrm{~W}_{\mathrm{k}}$. For both NOMA and OFDMA, the sum throughput or the sum capacity can be calculated by

$$
\mathrm{R}_{\mathrm{T}}=\sum_{\mathrm{k}=1}^{\mathrm{K}} \mathrm{R}_{\mathrm{k}}
$$

From the above equations, the sum throughput of both NOMA and OFDMA systems can be calculated. The Sum throughput is the sum of the data rates that are sent to all the users in a network. The Sum rate or Sum throughput value does not contribute anything to the fairness measures of the users data rate. Hence the Fairness measures or metrics play vital role in determining whether users or devices share a fair share of data rate. For calculating Fairness index for both NOMA and OFDMA, it can be defined as follows.

$$
\mathrm{F}=\frac{\left(\sum R_{k}\right)^{2}}{K \sum R_{k}^{2}}
$$

\section{SIMULATION RESULTS AND DISCUSSION}

In this section, the performance of Sum-throughput and Fairness-index of Non-orthogonal multiple access system is evaluated and it is then compared with the Sum-throughput and Fairness-index of orthogonal multiple access system on justifying the importance of NOMA to support effective spectrum efficiency and massive connectivity of M2M devices. The following parameters with values are considered to evaluate the aimed performance of the both NOMA and OFDMA. For the simulation purpose, a single BS with two users are considered. The channel gains corresponding to both UE1 and UE2 are assigned the values $\left(\mathrm{g}_{1}^{2}=-120 \mathrm{~dB}\right.$ and $\left.\mathrm{g}_{2}^{2}=-140 \mathrm{~dB}\right)$. Noise density N0 value is taken as $150 \mathrm{dBW} / \mathrm{Hz}$. The Base station static power is assigned to be $100 \mathrm{~W}$.

In the simulation graphs, the Sum-throughput is calculated for both the users and plotted for different system bandwidth values $(1.4 \mathrm{MHz}, 3 \mathrm{MHz}, 5 \mathrm{MHz}, 10 \mathrm{MHz}$ and $20 \mathrm{MHz})$. The graphs are plotted for Non-orthogonal multiple access and conventional OFDMA systems.

From the figure 3, the sum-throughput or the sum capacity is plotted with respect to users allocation power for the considered system bandwidth of $1.4 \mathrm{MHz}$. It is quite true from the figure, that the sum-throughput of NOMA is better than that of OFDMA system. For low power values of both NOMA and OFDMA, the increase of sum-throughput is more steep which is the quite interesting need of Machine-to-Machine Communication Devices. For other power values after the steep curve, the increase of sum-throughput is slow and gradual. Figure 4 shows the sum-throughput analysis for system bandwidth value equals to $3 \mathrm{MHz}$. Figures 5 and 6 exhibit the sum throughput analysis for the bandwidth value of $5 \mathrm{MHz}$ and $10 \mathrm{MHz}$ respectively. From all the plots, it can be witnessed that increase in system bandwidth increases the sum capacity of users and for lower power values, increasing in sum-throughput is notable due to steep rise which suits for $\mathrm{M} 2 \mathrm{M}$ communication. For other system bandwidth values such as $15 \mathrm{MHz}$ and $20 \mathrm{MHz}$, analysis graphs are shown in figures 7 and 8 .

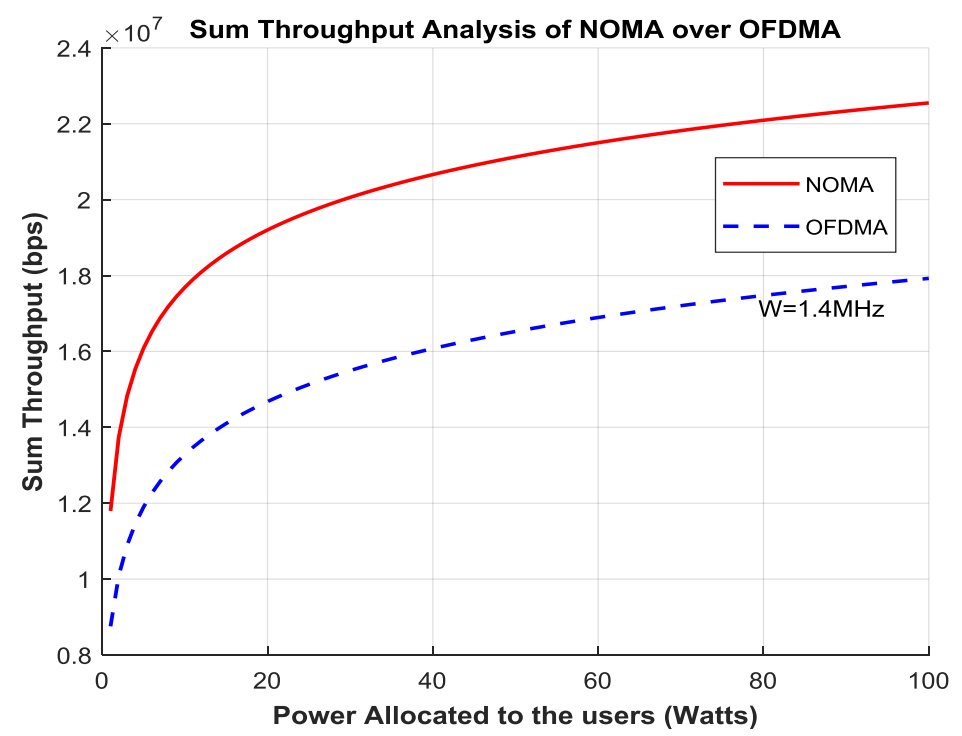

Fig. 3 Sum-throughput analysis of OFDMA and NOMA when system bandwidth $=1.4 \mathrm{MHz}$ 
International Journal of Advanced Research in Computer and Communication Engineering

Vol. 8, Issue 5, May 2019

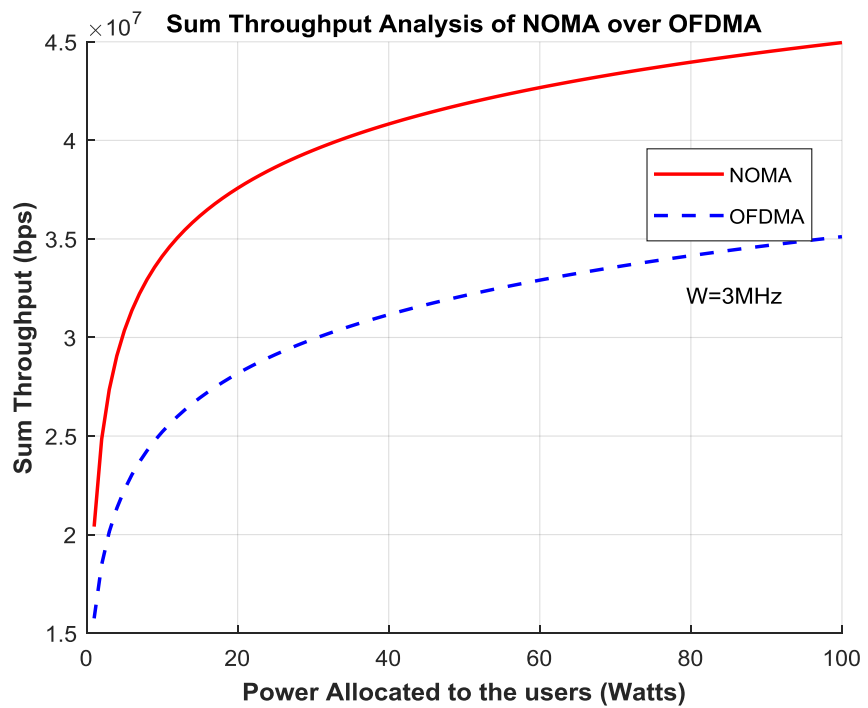

Fig. 4 Sum-throughput analysis of OFDMA and NOMA when system bandwidth $=3 \mathrm{MHz}$

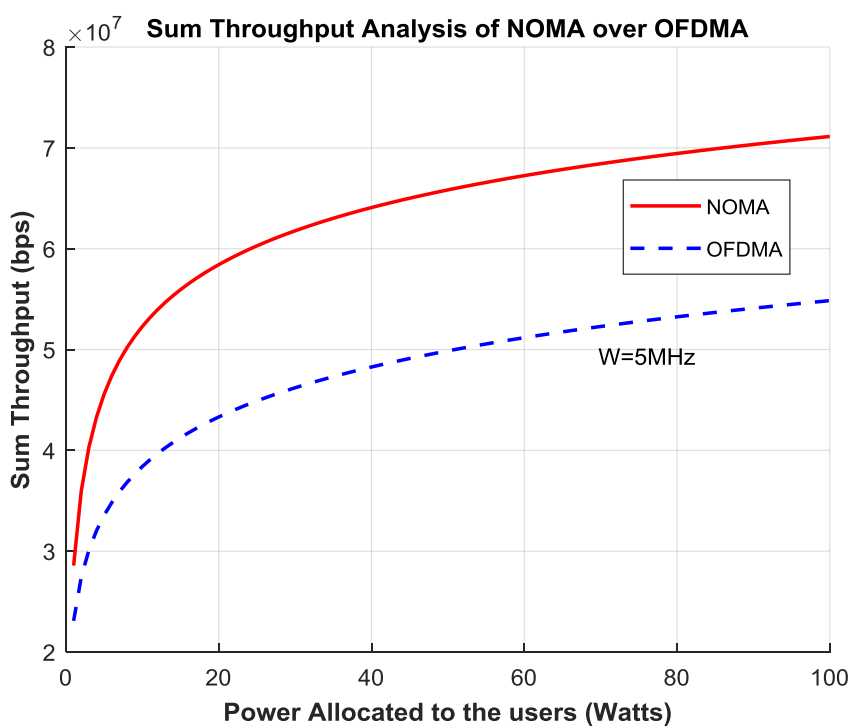

Fig. 5 Sum-throughput analysis of OFDMA and NOMA when system bandwidth $=5 \mathrm{MHz}$

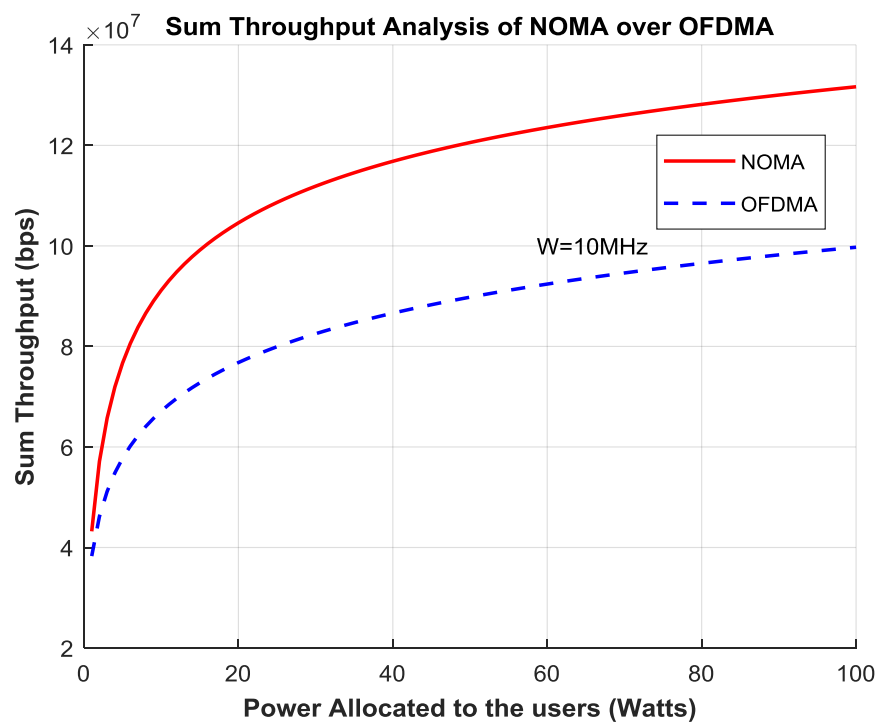

Fig. 6 Sum-throughput analysis of OFDMA and NOMA when system bandwidth $=10 \mathrm{MHz}$ 
Vol. 8, Issue 5, May 2019

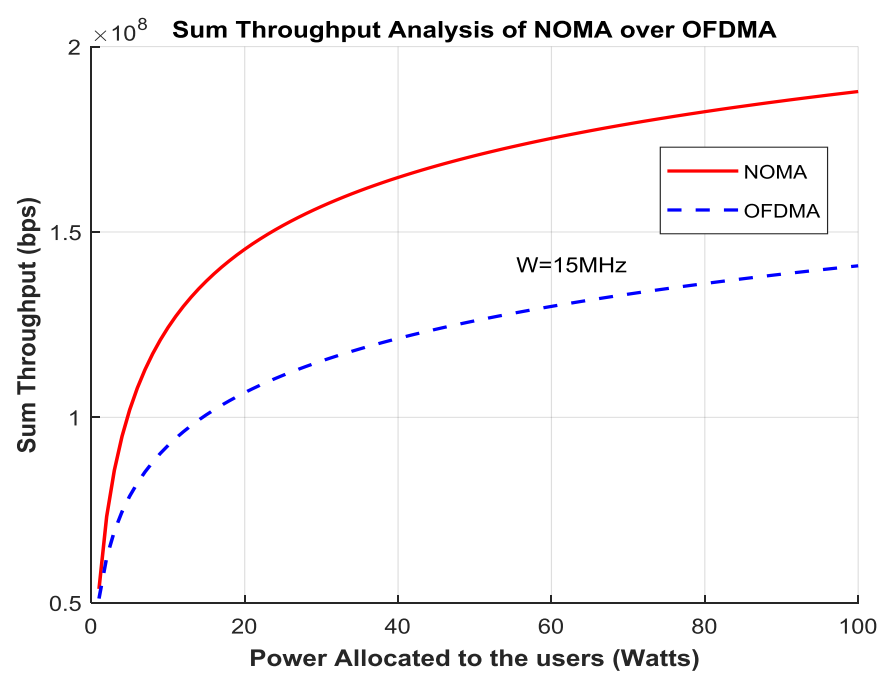

Fig. 7 Sum-throughput analysis of OFDMA and NOMA when System Bandwidth $=15 \mathrm{MHz}$

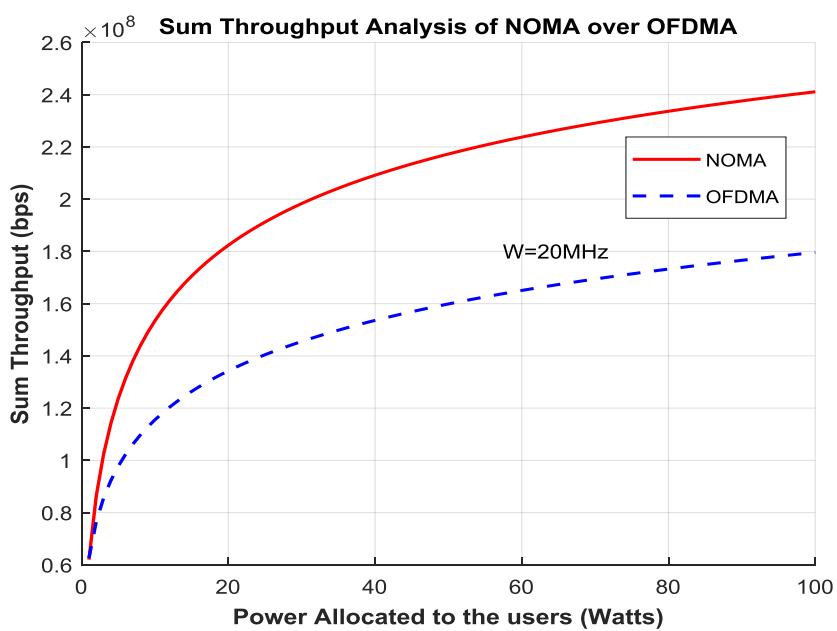

Fig. 8 Sum-throughput analysis of OFDMA and NOMA when System Bandwidth $=20 \mathrm{MHz}$

Figure 9 represents the rate pair analysis of NOMA and OFDMA. This analysis is considered for symmetric downlink channel. As clearly depicted in the given figure, NOMA achieves higher rate pairs than that of OFDMA. But at the corner points, the rates are equal to the single user capacities. With respect to fairness point of view, both users possess 1.6bps/Hz throughputs for both NOMA and OFDMA when the fairness index is higher. Sum throughput and individual throughputs stands good for NOMA for lower fairness index value.

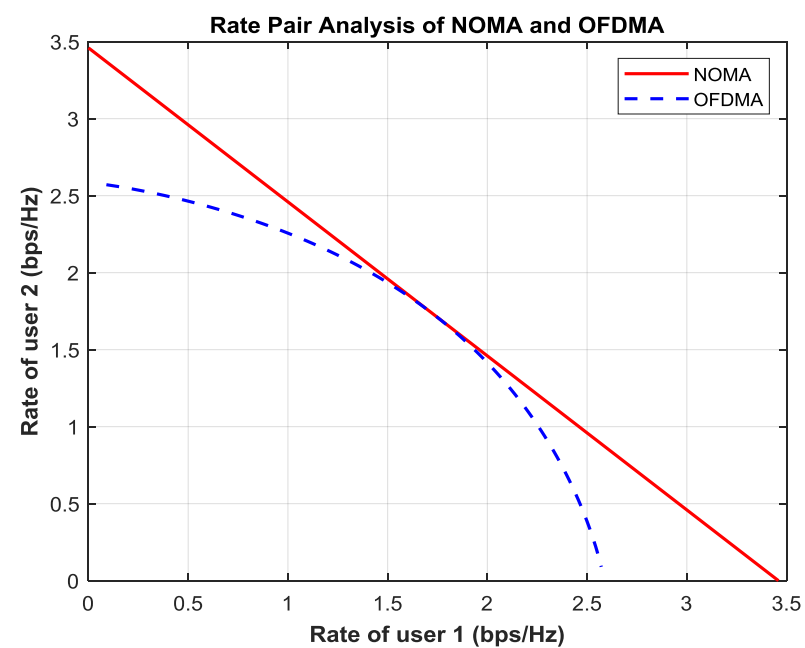

Fig. 9 Rate Pair analysis of NOMA and OFDMA 
Vol. 8, Issue 5, May 2019

Figure 10 shows the fairness index analysis of NOMA with respect of Power splitting factor (Alpha). When the power splitting factor is lower around 0.2 percent, Fairness index of NOMA reaches one and it clearly proves that information rate sharing among users stands good. Hence for NOMA, users in group are allocated with more difference of power between them and its Fairness index value is more suitable for Machine to Machine communication. Nearer users are allocated with lesser powers and far users are allocated with higher powers. Figure 11 shows the fairness index analysis of OFDMA with respect of Bandwidth splitting factor(Alpha). Here the fairness index value reaches one when the bandwidth is divided equally among users.

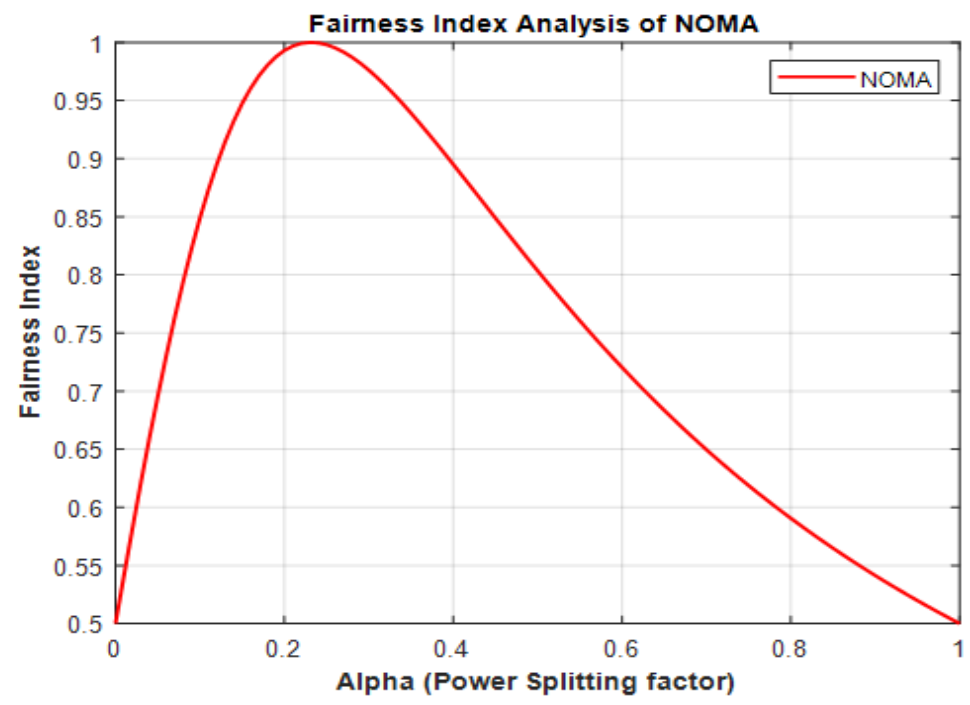

Fig. 10 Fairness Index Analysis of NOMA with respect to Power splitting factor

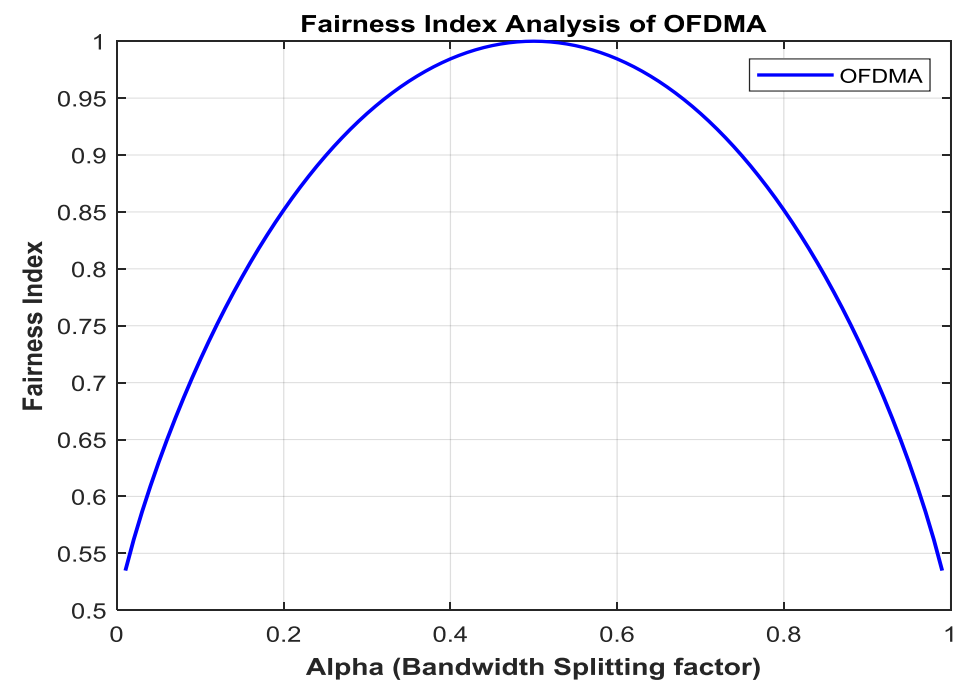

Fig. 11 Fairness Index Analysis of OFDMA with respect to Bandwidth splitting factor

It is quite obvious from all the figures that the NOMA system is superior to OFDMA system and achieves higher sum throughput and excellent fairness index value for supporting M2M devices. NOMA technique evidently outperforms the OFDMA system in order to achieve higher spectral efficiency and massive connectivity of devices and users.

\section{CONCLUSION}

In this paper, we have examined the performance analysis of the Sum-throughput and Fairness index of Non-orthogonal multiple access and compared in with the Sum-throughput and Fairness index of conventional orthogonal multiple access, From the simulation performance metrics, it can be witnessed and proved that the NOMA technique has yielded excellent result in terms of satisfying the criteria of users throughput and Fairness index over existing orthogonal multiple access which is the most ultimate need of massive numbers of users accessing the network in M2M communications. The overall performance of Non orthogonal multiple access system is superior to that of conventional 
OFDMA with respect to Sum-throughput and Fairness index and thus, NOMA is proved to be a potential technology for future mobile communications such as $5 \mathrm{G}$ to accommodate and serve the massive increase in the number of $\mathrm{M} 2 \mathrm{M}$ communication devices.

\section{REFERENCES}

[1]. Cisco: 'Cisco Visual Networking Index: global mobile data traffic forecast update, 2015 - 2020'. White Paper, 2016.

[2]. J. Andrews, S. Buzzi, W. Choi, S. Hanly, A. Lozano, A. Soong, and J. Zhang, "What will 5G be?" IEEE J. Select. Areas

[3]. Commun., vol. 32, no. 6, pp. 1065-1082, June 2014.

[4]. V.W.S. Wong, R. Schober, D.W.K. Ng, and L. C. Wang, Key Technologies for 5G Wireless Systems. Cambridge University Press, 2017.

[5]. Z. Ding et al., “Application of non-orthogonal multiple access in LTE and 5G networks," IEEE Commun. Mag., vol. 55, no. 2, pp. 185_191, Feb. 2017.

[6]. G. Wunder, P. Jung, M. Kasparick, T. Wild, F. Schaich, Y. Chen,S. Ten Brink, I. Gaspar, N. Michailow, A. Festaget al., "5GNOW: non orthogonal, asynchronous waveforms for future mobile applications," IEEE Commun. Mag., vol. 52, no. 2, pp. 97-105, Feb. 2014.

[7]. J.-B. Seo, B. C. Jung, and H. Jin, "Non-orthogonal random access for 5G mobile communication systems," IEEE Trans. Veh. Technol, vol. 67, no. 8, pp. 7867-7871, Aug. 2018.

[8]. L. Dai, B. Wang, Y. Yuan, et al., "Non-orthogonal multiple access for 5G: Solutions, challenges, opportunities, and future research

[9]. trends," IEEE Commun Magazine, vol. 53, no. 9, pp. 74-81, Sep. 2015.

[10]. Y. Saito, Y. Kishiyama, A. Benjebbour, T. Nakamura, A. Li, and K. Higuchi, “Non-orthogonal multiple access (NOMA) for cellular future radio access," in Proc. IEEE VTC-Spring, Dresden, Germany, Jun. 2013, pp. 1_5.

[11]. K. Higuchi and A. Benjebbour, "Non-orthogonal multiple access (NOMA) with successive interference cancellation for future radio access," IEICE Trans. Commun., vol. E98_B, no. 3, pp. 403_414, Mar. 2015.

[12]. Z. Ding, Z. Yang, P. Fan, and H. V. Poor, 'On the performance of non-orthogonal multiple access in 5G systems with randomly deployed users," IEEE Signal Process. Lett., vol. 21, no. 12, pp. 1501_1505, Dec. 2014.

[13]. Z. Yang, Z. Ding, P. Fan, and G. K. Karagiannidis, “'On the performance of non-orthogonal multiple access systems with partial channel information," IEEE Trans. Commun., vol. 64, no. 2, pp. 654_667, Feb. 2016.

[14]. Y. Saito, A. Benjebbour, Y. Kishiyama, and T. Nakamura, “'System-level performance evaluation of downlink non-orthogonal multiple access (NOMA)," in Proc. IEEE Int. Symp. Pers., Indoor Mobile Radio Commun. (PIMRC), Sep. 2013, pp. $611 \_615$.

[15]. X. Yue, Y. Liu, S. Kang and A. Nallanathan, "Performance analysis of NOMA with fixed gain relaying over Nakagami-m fading channels," IEEE Access, vol. 5, no. , pp. 5445-5454, 2017.

[16]. N. Otao, Y. Kishiyama, and K. Higuchi, "Performance of non-orthogonal access with SIC in cellular downlink using proportional fair-based resource allocation," in Proc. IEEE Intern. Sympos. on Wireless Commun. Systems, Aug. 2012, pp. 476-480.

[17]. M. Zou, S. Chan, H. Vu, and L. Ping, “Throughput improvement of 802.11 networks via randomization of transmission power levels," IEEE Trans. Veh. Technol., vol. 65, no. 4, pp. 2703_2714, Apr. 2016.

[18]. M. F. Hanif, Z. Ding, T. Ratnarajah, and G. K. Karagiannidis, "A minorization-maximization method for optimizing sum rate in the downlink of non-orthogonal multiple access systems,” IEEE Trans. Signal Process., vol. 64, no. 1, pp. 76-88, Jan. 2016.

[19]. Y. Zhang, H. M. Wang, Q. Yang, and Z. Ding, "Secrecy sum rate maximization in non-orthogonal multiple access,” IEEE Commun. Lett., vol. PP, no. 99, pp. 1-1, Mar. 2016.

[20]. J. Umehara, Y. Kishiyama, and K. Higuchi, “Enhancing user fairness in non-orthogonal access with successive interference cancellation for cellular downlink," in Proc. IEEE ICCS, Munich, Germany, Nov. 2012, pp. 324_328.

[21]. Z. Ding, P. Fan, and H. V. Poor, “'Impact of user pairing on 5G nonorthogonal multiple access," IEEE Trans. Veh. Technol., vol. 65, no. 8, pp. 6010_6023, Aug. 2016.

[22]. S. Timotheou and I. Krikidis, "Fairness for non-orthogonal multiple access in 5G systems," IEEE Signal Process. Lett., vol. 22, no. 10,

[23]. pp. 1647-1651, Oct. 2015.

[24]. A. Pastore and M. Navarro, “A fairness-throughput trade-off perspective on NOMA multiresolution broadcasting," IEEE Trans. Broadcast., pp. 1_9, Nov. 2018 . 